\title{
EIGENVALUES OF 2-TRIDIAGONAL TOEPLITZ MATRIX
}

\author{
Jolanta Borowska, Lena Lacińska \\ Institute of Mathematics, Czestochowa University of Technology \\ Czestochowa, Poland \\ jolanta.borowska@im.pcz.pl,lena.lacinska@im.pcz.pl
}

\begin{abstract}
In this article an explicit formula for eigenvalues of a 2-tridiagonal Toeplitz matrix can be derived on the basis of a certain relation between the determinant of this matrix and the determinant of a pertinent tridiagonal matrix. It can be pointed out that the problem is investigated without imposing any conditions on the elements of matrix.
\end{abstract}

Keywords: eigenvalues, $k$-tridiagonal matrices, Toeplitz matrix

\section{Introduction}

Numerical solutions of many problems of physics, statistics or signal processing involve the eigenvalue problems for $k$-tridiagonal matrices.

The eigenvalue problem for a tridiagonal Toeplitz matrix can be found in [1]. The determinant and inversion of $k$-tridiagonal matrices were presented for example in $[2,3]$.

The general $k$-tridiagonal matrix $A_{n}^{(k)}$ can be written in the form

$$
A_{n}^{(k)}=\left[a_{i j}\right]_{n \times n}
$$

where

$$
a_{i j}=\left\{\begin{array}{cc}
a_{i}, & i=j \\
b_{j}, & i-j=k \\
c_{i}, & j-i=k \\
0, & \text { otherwise }
\end{array}\right.
$$

The aim of this paper is to show that eigenvalues of a 2-tridiagonal Toeplitz matrix, which is the special case of general $k$-tridiagonal matrix, can be expressed by means of eigenvalues of pertinent tridiagonal Toeplitz matrices.

In the current paper the explicit formulae for eigenvalues of tridiagonal Toeplitz matrix can be obtained directly from the characteristic equation. Such approach enables us to derive eigenvalues of 2-tridiagonal matrix. 


\section{Eigenvalues of tridiagonal Toeplitz matrix}

Let us consider the eigenvalue problem for a tridiagonal Toeplitz matrix of the form

$$
A_{n}=\left[\begin{array}{ccccccc}
a & c & & & & & \\
b & a & c & & & & \\
& b & a & c & & & \\
& & \ddots & \ddots & \ddots & & \\
& & & \ddots & \ddots & \ddots & \\
& & & & b & a & c \\
& & & & & b & a
\end{array}\right]_{n \times n}
$$

It can be noted that the tridiagonal matrix (2) is a special case of $k$-tridiagonal matrix (1), when $k=1$.

Let $\lambda$ be an eigenvalue and $v=\left[v_{1}, \ldots, v_{n}\right]^{\mathrm{T}} \neq 0$ a corresponding eigenvector. Hence the eigenvalue problem for matrix $A_{n}$ has the form

$$
A_{n} v=\lambda v
$$

The above equation can be presented in the form

$$
\left(A_{n}-\lambda I_{n}\right) v=\mathbf{0}
$$

where $I_{n}$ is the identity matrix of an order $n$ and $\mathbf{0}$ is zero column matrix. Eigenvector $v$ is assumed to be nonzero, hence $\lambda$ is an eigenvalue of matrix $A_{n}$ if and only if

$$
\operatorname{det}\left(A_{n}-\lambda I_{n}\right)=0
$$

It can be seen that matrix $A_{n}-\lambda I_{n}$ is a tridiagonal Toeplitz matrix.

Let us denote $\operatorname{det}\left(A_{n}-\lambda I_{n}\right)$ by $W_{n}$. Bearing in mind [4], we obtain a recurrence relation for $W_{n}$

$$
W_{n+2}-(a-\lambda) W_{n+1}+b c W_{n}=0, \quad n \geq 1
$$

where

$$
W_{1}=a-\lambda, \quad W_{2}=(a-\lambda)^{2}-b c
$$

The solution to equation (6) with initial conditions (7) depends on $\Delta=(a-\lambda)^{2}-4 b c$. If $\Delta \neq 0$ then, cf. [4], we have 


$$
W_{n}=\frac{1}{\sqrt{\Delta}}\left(p^{n+1}-q^{n+1}\right)
$$

where

$$
\begin{aligned}
& p=\frac{a-\lambda+\sqrt{(a-\lambda)^{2}-4 b c}}{2} \\
& q=\frac{a-\lambda-\sqrt{(a-\lambda)^{2}-4 b c}}{2}
\end{aligned}
$$

Hence, condition (5) holds if

$$
p^{n+1}-q^{n+1}=0
$$

Let us observe that

$$
p \cdot q=b c
$$

Bearing in mind (11), equation (10) can be converted to

$$
\left(\frac{p^{2}}{b c}\right)^{n+1}=1
$$

The above condition together with (11) leads to two cases for $p$ and $q$

$$
\begin{aligned}
& p=\sqrt{b c}\left(\cos \frac{s \pi}{n+1}+i \sin \frac{s \pi}{n+1}\right), \quad s=1,2, \ldots,(n+1) \\
& q=\sqrt{b c}\left(\cos \frac{s \pi}{n+1}-i \sin \frac{s \pi}{n+1}\right), \quad s=1,2, \ldots,(n+1)
\end{aligned}
$$

or

$$
\begin{array}{ll}
p=-\sqrt{b c}\left(\cos \frac{s \pi}{n+1}+i \sin \frac{s \pi}{n+1}\right), & s=1,2, \ldots,(n+1) \\
q=-\sqrt{b c}\left(\cos \frac{s \pi}{n+1}+i \sin \frac{s \pi}{n+1}\right), & s=1,2, \ldots,(n+1)
\end{array}
$$

It can be seen that (12) and (13) lead to the same resulting eigenvalues. Hence, subsequently we consider only case given by relations (12).

From (12) we have

$$
p+q=2 \sqrt{b c} \cos \frac{s \pi}{n+1}, \quad s=1,2, \ldots,(n+1)
$$


At the same time from (9) we have

$$
p+q=a-\lambda
$$

Bearing in mind (14) and (15), we obtain

$$
a-\lambda=2 \sqrt{b c} \cos \frac{s \pi}{n+1}, \quad s=1,2, \ldots,(n+1)
$$

Let us observe that when $s=n+1$ we have $\lambda=a+2 \sqrt{b c}$, so condition $\Delta \neq 0$ doesn't hold for any $a, b, c$. Therefore, in this case eigenvalues $\lambda_{s}, s=1, \ldots, n$ of matrix $A_{n}$ have the form

$$
\lambda_{s}=a-2 \sqrt{b c} \cos \frac{s \pi}{n+1}, s=1,2, \ldots, n
$$

Let us observe that eigenvalues $\lambda_{s}, s=1, \ldots, n$ given by (17) satisfy condition $\Delta \neq 0$ when $b \neq 0$ and $c \neq 0$.

Now, let us assume that $\Delta=0$. In this case the solution to equation (6) with initial conditions (7) have the form, cf. [4]

$$
W_{n}=\frac{2 b c+n\left((a-\lambda)^{2}-2 b c\right)}{2}\left(\frac{a-\lambda}{2}\right)^{n-2}
$$

Hence, condition (5) holds if

$$
2 b c+n\left((a-\lambda)^{2}-2 b c\right)=0
$$

or

$$
\left(\frac{a-\lambda}{2}\right)^{n-2}=0
$$

From (19) we have

$$
\lambda_{1}=a-\sqrt{\frac{2 b c(n-1)}{n}}, \quad \lambda_{2}=a+\sqrt{\frac{2 b c(n-1)}{n}}
$$

At the same time, from (20) we have

$$
\lambda_{s}=a, \quad s=3, \ldots, n
$$


Let us observe that $\lambda_{s}, s=1, \ldots, n$ given by (21) and (22) satisfy condition $\Delta=0$ when $b=0$ or $c=0$. So relations (21) and (22) reduce to

$$
\lambda_{s}=a, \quad s=1, \ldots, n
$$

It can be seen that relation (23) can be obtained from (17) under assumption $b=0$ or $c=0$. So, we conclude that all eigenvalues of tridiagonal matrix (2) can be expressed by formula (17) and there is no need to take any assumptions on elements of the matrix under considerations.

\section{Eigenvalues of a 2-tridiagonal Toeplitz matrix}

In this section we consider a 2-tridiagonal Toeplitz matrix, which is obtained from (1) under assumption that $k=2$ and $a_{i}=a, i=1, \ldots, n, b_{j}=b, j=1, \ldots, n-1$, $c_{l}=c, l=1, \ldots, n-1$. So this matrix has the form

$$
A_{n}^{(2)}=\left[\begin{array}{ccccccc}
a & 0 & c & & & & \\
0 & a & 0 & c & & & \\
b & 0 & a & 0 & c & & \\
& & \ddots & \ddots & \ddots & \ddots & \\
& & & \ddots & \ddots & \ddots & c \\
& & & b & 0 & a & 0 \\
& & & & b & 0 & a
\end{array}\right]_{n \times n}
$$

The eigenvalue problem for matrix (24) has the form

$$
A_{n}^{(2)} v=\lambda v
$$

It means that $\lambda$ is an eigenvalue of matrix (24) if and only if

$$
\operatorname{det}\left(A_{n}^{(2)}-\lambda I_{n}\right)=0
$$

Let us denote $\operatorname{det}\left(A_{n}^{(2)}-\lambda I_{n}\right)$ by $W_{n}^{(2)}$. It can be proved, [5], that

$$
W_{n}^{(2)}= \begin{cases}\left(W_{\frac{n}{2}}\right)^{2}, & n \text { is even } \\ W_{\frac{n-1}{2}} W_{\frac{n+1}{2}}, & n \text { is odd }\end{cases}
$$


where $W_{\frac{n}{2}}, W_{\frac{n-1}{2}}, W_{\frac{n+1}{2}}$ are the determinants of tridiagonal matrices of the form as matrix (2) of orders $\frac{n}{2}, \frac{n-1}{2}$ and $\frac{n+1}{2}$, respectively.

Case 1.

Let us assume that matrix (24) has the even order $n$.

In this case condition (26) has the form

$$
\left(W_{\frac{n}{2}}\right)^{2}=0
$$

Bearing in mind all considerations from section 2, we obtain from (28) that 2-tridiagonal matrix (24) of the even order $n$ has $\frac{n}{2}$ different eigenvalues, which have the form

$$
\lambda_{s}=a-2 \sqrt{b c} \cos \frac{2 s \pi}{n+2}, s=1, \ldots, \frac{n}{2}
$$

Case 2.

Now, let us assume that matrix (24) has the odd order $n$.

In this case condition (26) leads to

$$
W_{\frac{n-1}{2}} W_{\frac{n+1}{2}}=0
$$

Condition (30) holds if

$$
W_{\frac{n-1}{2}}=0
$$

or

$$
W_{\frac{n+1}{2}}=0
$$

Bearing in mind all considerations given in section 2, we obtain from (31)

$$
\lambda_{s}=a-2 \sqrt{b c} \cos \frac{2 s \pi}{n+1}, s=1, \ldots, \frac{n-1}{2}
$$

and analogically from (32)

$$
\lambda_{s}=a-2 \sqrt{b c} \cos \frac{2 s \pi}{n+3}, s=1, \ldots, \frac{n+1}{2}
$$


Formulas (33) and (34) represent $n$ different eigenvalues of 2-tridiagonal matrix (24) of the odd order $n$.

\section{Conclusions}

It was shown that the well-known formula on eigenvalues of a tridiagonal Toeplitz matrix can be derived directly from the characteristic equation. Moreover, the formulas on eigenvalues of a 2-tridiagonal Toeplitz matrix were derived on the basis of the relations between the determinant of this matrix and pertinent tridiagonal Toeplitz matrices. It has to be emphasized that 2-tridiagonal Toeplitz matrix of the even order $n$ has $n / 2$ different eigenvalues, whereas the matrix of the odd order $n$ has $n$ different eigenvalues. All obtained results don't depend on elements of matrices under considerations.

\section{References}

[1] Yueh W.C., Eigenvalues of tridiagonal matrices, Applied Mathematics E-Notes 2005, 5, 66-74.

[2] Asci M., Tasci D., El-Mikkawy M., On determinants and permanents of k-tridiagonal Toeplitz matrices, Util. Math. 2012, 89, 97-106.

[3] Jia J., Sogabe T., El-Mikkawy M., Inversion of k-tridiagonal matrices with Toeplitz structure, Computers and Mathematics with Applications 2013, 65, 116-125.

[4] Borowska J., Łacińska L., Rychlewska J., Application of difference equation to certain tridiagonal matrices, Scientific Research of the Institute of Mathematics and Computer Science 2012, $3(11), 15-20$.

[5] Borowska J., Lacińska L., Rychlewska J., On determinant of certain pentadiagonal matrix, Journal of Applied Mathematics and Computational Mechanics 2013, 12(3), 21-26. 